\title{
Influence of droplet size on the growth of high-quality self-catalyzed GaAsP nanowires
}

Yunyan Zhang

Ana M. Sanchez

Martin Aagesen

Yue Sun

Jiang $\mathrm{Wu}$

Dongyoung Kim

Pamela Jurczak

Suguo Huo

Xiulai Xu

Huiyun Liu 


\title{
Influence of Droplet Size on the Growth of High-Quality Self- Catalyzed GaAsP Nanowires
}

Yunyan Zhang*a, Ana M. Sanchez ${ }^{\text {, }}$, Martin Aagesen ${ }^{c}$, Yue Sun ${ }^{\mathrm{d}}$, Jiang Wu $\mathrm{Wu}^{\mathrm{a}}$, Dongyoung Kim a, Pamela Jurczak ${ }^{\text {a }}$, Suguo Huo ${ }^{\mathrm{e}}$, Xiulai Xu ${ }^{\mathrm{d}}$, Huiyun Liu ${ }^{\mathrm{a}}$

${ }^{a}$ Department of Electronic and Electrical Engineering, University College London, London WC1E 7JE, United Kingdom;

${ }^{\mathrm{b}}$ Department of Physics, University of Warwick, Coventry CV4 7AL, United Kingdom

${ }^{c}$ Gasp Solar ApS, Gregersensvej 7, Taastrup DK-2630, Denmark;

d Beijing National Laboratory for Condensed Matter Physics, Institute of Physics, Chinese Academy of Sciences, Beijing 100190, People's Republic of China

${ }^{\mathrm{e}}$ London Centre for Nanotechnology, University College London, London WC1H 0AH, United Kingdom

\begin{abstract}
Nanowires (NWs) have better functionality and superior performance as compared with the traditional thin film counterparts. However, NW growth is highly complicated and the growth mechanism is far from clear, especially when it is grown by vapor-liquid-solid mode. In this work, the influences of droplet size on the growth of self-catalyzed ternary NWs were studied using GaAsP NWs. The size-induced Gibbs-Thomson (GT) effect is observed for the first time in the self-catalyzed growth mode, which can make the smaller catalytic droplets have lower effective supersaturations. Thus, the droplet size can significantly influence the uniformity and composition of NWs. By carefully control the droplet size, the growth of highly uniform NW arrays are demonstrated. These results provide useful information for understanding the mechanisms of self-catalyzed III-V NW nucleation and growth with the important ternary III-V material systems.
\end{abstract}

Keywords: Nanowire, droplet size, self-catalyzed, Gibbs-Thomson effect, GaAsP.

\section{INTRODUCTION}

Nanowires (NWs) with a one-dimensional (1D) columnar shape at nanometer scale can lead to different crystallographic, photonic, electrical, and mechanical properties than those of their thin film counterparts. ${ }^{1-7}$ These advantages can give new theories for device structure design. ${ }^{8,9}$ For example, the nanoscale cross-section can efficiently relax strain without degrading the crystal quality of NWs. Thus, the limitations, caused by the requirement of lattice and thermal expansion coefficient, ${ }^{10-13}$ can be greatly alleviated, giving more freedom in the material combination and the substrate choice, e.g. monolithically integrating III-Vs on $\mathrm{Si}^{14-17}$

Droplet catalyzed mode, also called vapor-liquid-solid (VLS) mode, is one of the most popular NW growth mode. It is significantly different from the traditional thin film growth using the vapor-solid mode. During the growth with this mode, a catalytic droplet is needed for each NW. All the materials (except the sidewall growth) need to dissolve into the catalytic droplet and then incorporate into NWs driven by droplet supersaturation. Thus, the catalytic droplet is playing a leading role. Over decades, great effort from researchers has been put on Au-catalyzed NW growth. However, the function and influence of the catalytic droplet are still far from clear. Recent years, the self-catalyzed growth mode is taking over the foreign-metal ( $\mathrm{Au}$ ) catalyzed growth mode. This is because the material of the catalytic droplet is also one element of NWs, e.g. Ga droplet for GaAs NWs, which can avoid the foreign metal contamination (e.g. Au) and have better control over NW shell growth. However, due to the consumable feature of the catalytic droplet, the NW growth mechanism is highly complicated and the growth is extremely difficult to control. ${ }^{18}$

In this work, the influence of catalytic droplet on the growth of self-catalyzed NWs are studied using GaAsP NWs. We observed, for the first time, the Gibbs-Thomson (GT) effect in the self-catalyzed growth mode. Due to this effect, NWs grown from different droplet size exhibit different growth rates and P contents. By careful droplet size control, we demonstrated the growth of highly uniform NW arrays. 


\section{EXPERIMENT}

The GaAsP NWs were grown on 380- $\mu$ m-thick p-doped silicon (111) substrates by a solid-source molecular beam epitaxy. There were two approaches used for the GaAsP NW growth. In the first approach, growth was initiated with a GaAs stem for 5 minutes and then followed by a 55-minute GaAsP growth, which are called GaAs-stem GaAsP NWs (GS-GaAsP NWs) for simplicity. The other growth method started directly with GaAsP and lasted for 1 hour, which is called directly-grown GaAsP NWs (DG-GaAsP NWs). If not indicated otherwise, the GaAs stem was grown with a Ga beam equivalent pressure of $1.12 \times 10^{-7}$ Torr and V/III flux ratio of 60 at $\sim 640{ }^{\circ} \mathrm{C}$. The GaAsP segments of both types of NWs were grown with a Ga beam equivalent pressure, $\mathrm{V} / \mathrm{III}$ flux ratio, $\mathrm{P} /(\mathrm{As}+\mathrm{P})$ flux ratio, and substrate temperature of $1.12 \times 10^{-7}$ Torr, 50, 0.12 , and $\sim 640^{\circ} \mathrm{C}$, respectively. GaAs NWs were grown with a Ga beam equivalent pressure, V/III flux ratio, substrate temperature, and growth duration of $1.12 \times 10^{-7}$ Torr, $44, \sim 630^{\circ} \mathrm{C}$ and 1 hour, respectively. The substrate temperature was measured by a pyrometer for the growth of core GaAsP nanowires. The patterned growth was on $\mathrm{Si}(111)$ substrates with the $\mathrm{SiO}_{2}$ patterned prepared using nanoimprint lithography. The NWs were grown at a temperature $\sim 630{ }^{\circ} \mathrm{C}$ for 45 min with a Ga flux of $1.6 \times 10-7$ Torr, V/III flux ratios between 3 and 20 , and a P/(P + As) flux ratio of $12 \%$. Further details can be found elsewhere ${ }^{19-21}$ The NW morphology was measured by a Zeiss XB 1540 focus ion beam/scanning electron microscopes (FIB/SEM) system. The transmission electron microscope (TEM) analysis was performed on JEOL 2100 and JEOL ARM200F microscopes operating at $200 \mathrm{kV}$. Compositional analysis using energy-dispersive X-ray spectrometry (EDX) was performed using Oxford Instruments $100 \mathrm{~mm}^{2}$ SDD EDX detectors. Power-dependent photoluminescence (PL) spectroscopy was measured by a confocal microphotoluminescence system with a laser spot diameter of $\sim 2 \mu \mathrm{m}$ and the spectra were detected with a liquid nitrogen cooled charge coupled device camera. Room-temperature PL measurement was performed using a nanometrics RPM2000 machine with excitation wavelength of $635 \mathrm{~nm}$ and power density of $\sim 500 \mathrm{~W} / \mathrm{cm}^{2}$.

\section{RESULTS AND DISCUSSION}

To obtain droplets of different sizes, NW growth was started with a 5-min GaAs stem growth (Figure 1). At the beginning of the growth, the clean substrate surface was beneficial for the formation of droplets with uniform diameters. However, because growth temperature was $5 \sim 10^{\circ} \mathrm{C}$ higher than conventional GaAs NW growth, the droplets for the GaAs stem growth were quite large and hence led to NWs with large diameters $(\sim 90 \mathrm{~nm})$. After the GaAs stem, the growth switched to the GaAsP growth. The introduction of the P flux for GaAsP growth significantly increased the chemical potential of the vapor phase, which can promote the formation of the second batch of droplets. Due to the strong competition and interference from the first-batch GaAs stems, the inferior Ga collection environment made the size of the second-batch droplets much smaller and non-uniform.

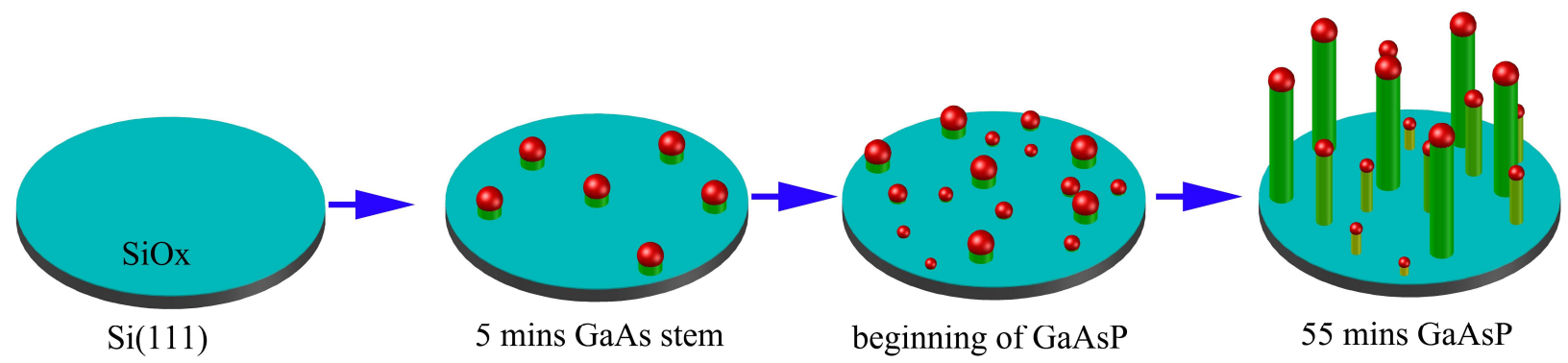

Figure 1. Illustration of the GS-GaAsP NWs growth by using different droplet size. 

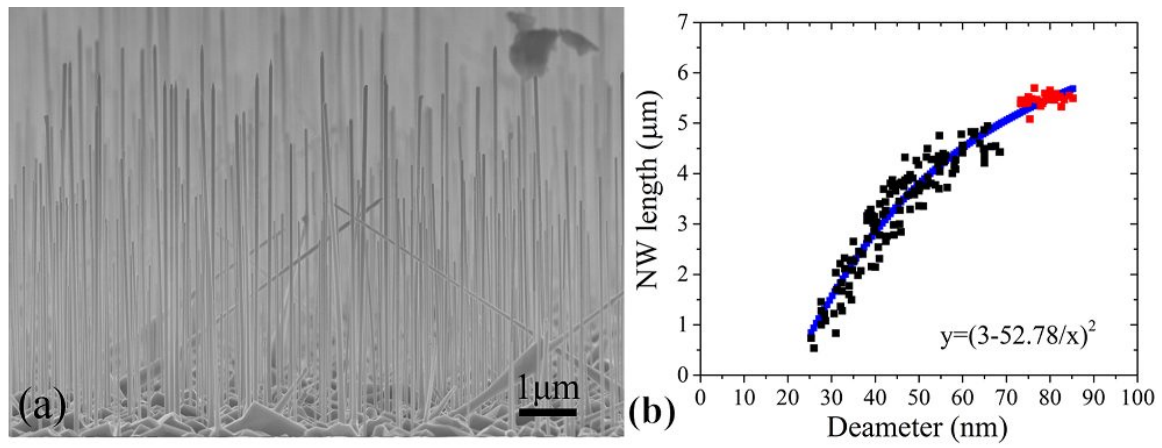

Figure 2. (a) Side view SEM image and (b) length-diameter summation of GS-GaAsP NWs grown at $\sim 635^{\circ} \mathrm{C}$. The blue line in (b) is the fitting curve described by the inset equation. The red dots in (b) are corrected by reducing the actual length by 5/60.

As can be seen in Figure 2, the lengths of GaAsP NWs vary significantly. This large length variation is connected with the NW diameter differences. As can be seen from the diameter-length summation in Figure $1 \mathrm{~b}$, the NWs with larger diameters are generally longer. The experiment data can be fitted well by Equation 1 with $\mathrm{A}=3$ and $\mathrm{B}=52.78$.

$$
E=\left(A-\frac{B}{6}\right)^{2}
$$

This can be explained by the GT effect that can increased the vapor pressure of smaller droplets more than that of the larger ones. Therefore, the effective supersaturation $\angle \beta$ (the effective difference between the chemical potentials of elements in the vapor phase and in the NW) inside the droplet with a smaller diameter is lower, which can be expressed as:

$$
A \mu=\Delta \mu_{0}=4 \Omega \alpha_{\mathrm{wg}} / d
$$

where the $\Delta \mu_{0}$ is the supersaturation in the planar limit (i.e., $\mathrm{d} \rightarrow \infty$ ), $\Omega$ is the atomic volume of the growth species, $\mathrm{d}$ is the diameter of the NW, and $\alpha_{v g}$ is the average surface energy density of the NW surface facets. Thus, the growth rate of smaller droplet becomes slower:

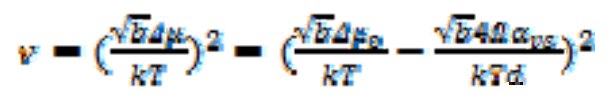

where $b$ is a kinetic coefficient of crystallization, $\mathrm{k}$ is Boltzmann's constant, and $\mathrm{T}$ is the temperature. Thus, the reduction of the $\Delta \mu$ can slow down the growth rate. With the growth time of t, the smaller NW is shorter in length, which can be expressed as:

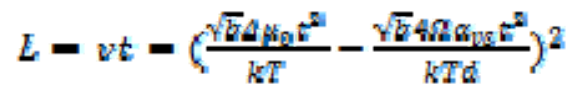

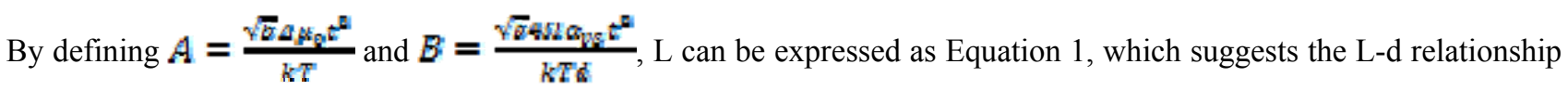
is controlled by GT effect. 


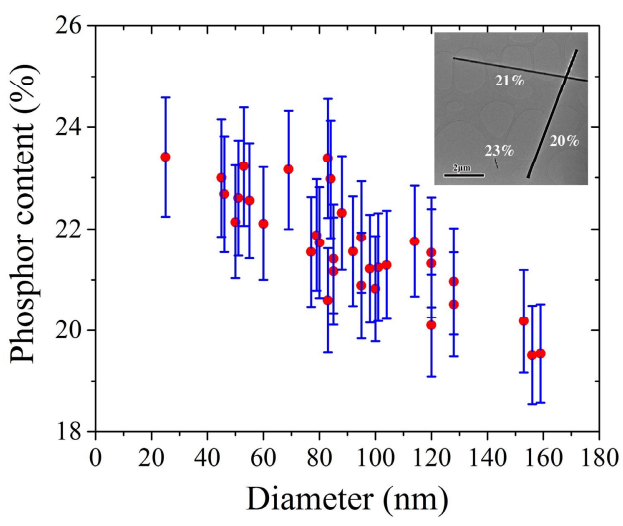

Figure 3. Phosphorus content VS NW diameter. Inset is phosphor content of GS-GaAsP NWs with three different diameters.

According to the GT effect, the smaller droplets should have higher vapor pressure and hence stronger out driving force to expel the group-V elements (As and P). P has a higher chemical potential with Ga in comparison to As, and hence stronger nucleation ability. This should make $\mathrm{P}$ more resistant to the out driving force as compared with As. To verify this, the composition of NWs with different diameter was measured. As shown in Figure 3, NWs with a smaller size have higher $\mathrm{P}$ content, which is consistent to the influence of GT effect.
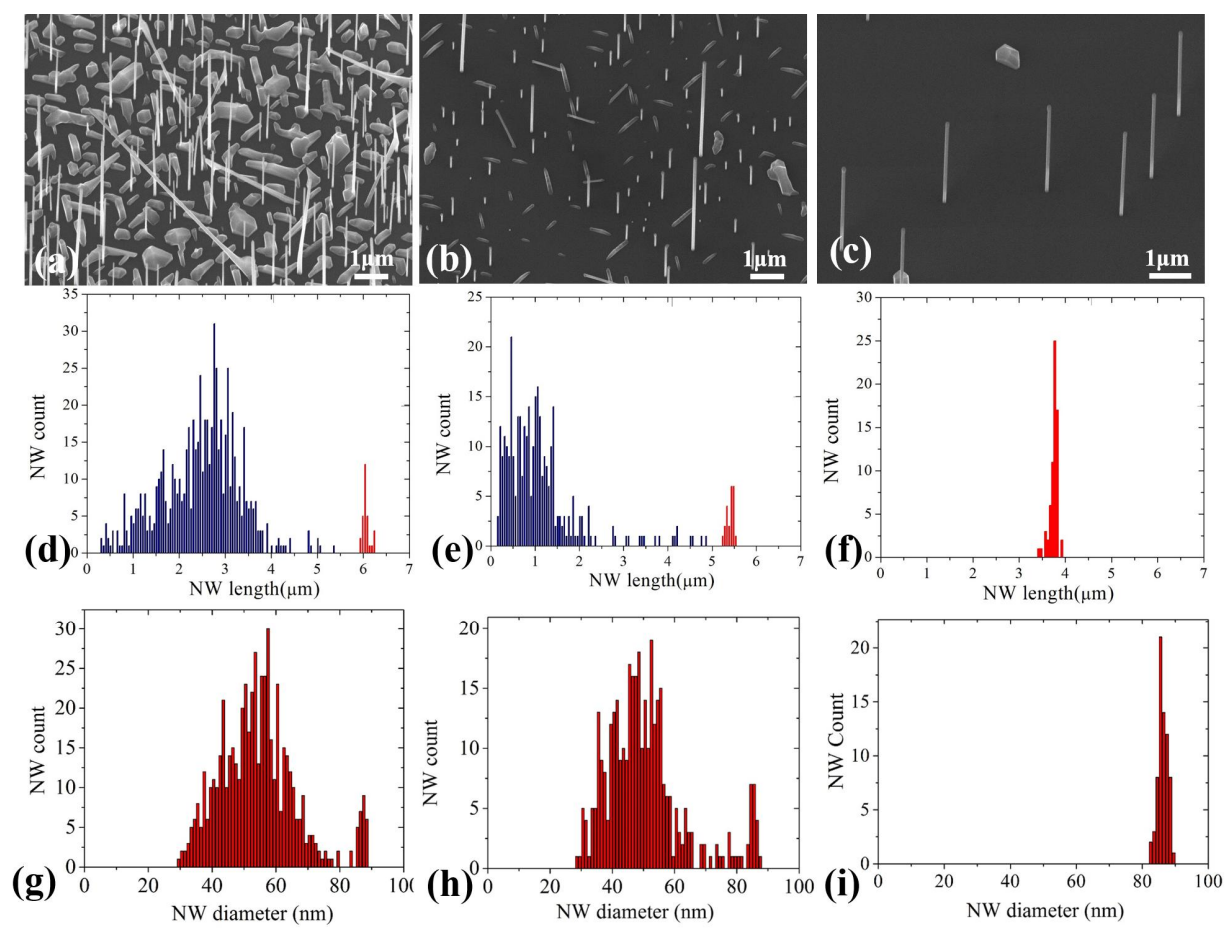

Figure $4.35^{\circ}$ tilted SEM image, length summation histogram, and diameter summation histogram of GS-GaAsP NWs grown at (a) (d) (g) $640^{\circ} \mathrm{C}$, (b) (e) (h) $\sim 645^{\circ} \mathrm{C}$, and (c) (f) (i) $\sim 650^{\circ} \mathrm{C}$.

To further confirm the existence of GT effect during NW growth, NWs were grown at different temperatures. At the growth temperature of $\sim 640^{\circ} \mathrm{C}$, the NWs can be roughly divided into two groups by their diameters and lengths, forming a bi-modal distribution (Figure $4 \mathrm{a}, \mathrm{d}$ and $\mathrm{g}$ ). With the increase of the growth temperature to $\sim 645^{\circ} \mathrm{C}$ (Figure $4 \mathrm{~b}$ ), the 
length reduction rate $\left(\frac{\Delta \mathrm{L}}{\mathrm{L}_{2}}\right.$ where $\Delta \mathrm{L}$ is the length reduction and $\mathrm{L}_{0}$ is the length of NWs) of the small-diameter group is much larger as compared to the NWs in the large-diameter group (Figure 4e). However, the size distribution of NWs did not change significantly, as can be seen in Figure $4 \mathrm{~h}$. Further increase of the growth temperature to $\sim 650^{\circ} \mathrm{C}$, the growth of small-diameter NWs was completely suppressed, leaving only large-diameter NWs with small density but good length and diameter uniformity, as shown in Figure 4c, $\mathrm{f}$ and i. The increase of growth temperature can decrease the supersaturation of droplets and hence reduce the NW growth rate. When the temperature is higher than the critical value, the supersaturation of the droplets can be too low to maintain the NW growth. For smaller droplets, the supersaturation is already lower than that of larger ones due to the influence of GT effect. Thus, their critical temperature is lower as compared with larger ones, making the small-diameter NW group disappear but large-diameter NW group can still grow at $\sim 650^{\circ} \mathrm{C}^{22}$

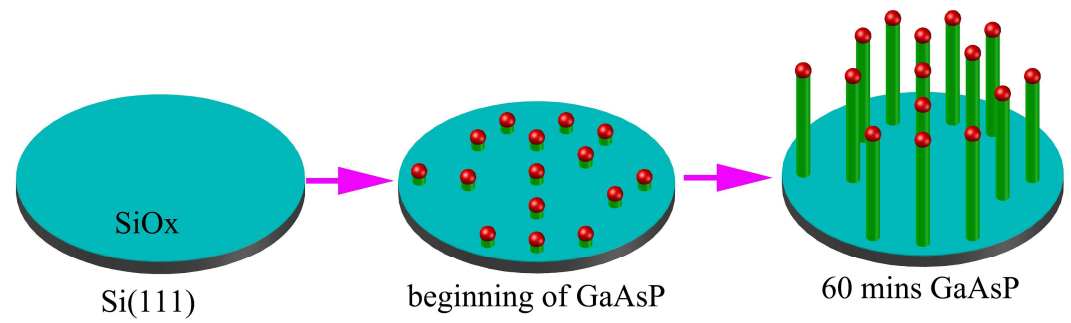

Figure 5. Illustration of the DG-GaAsP NWs growth by using uniform droplet size.
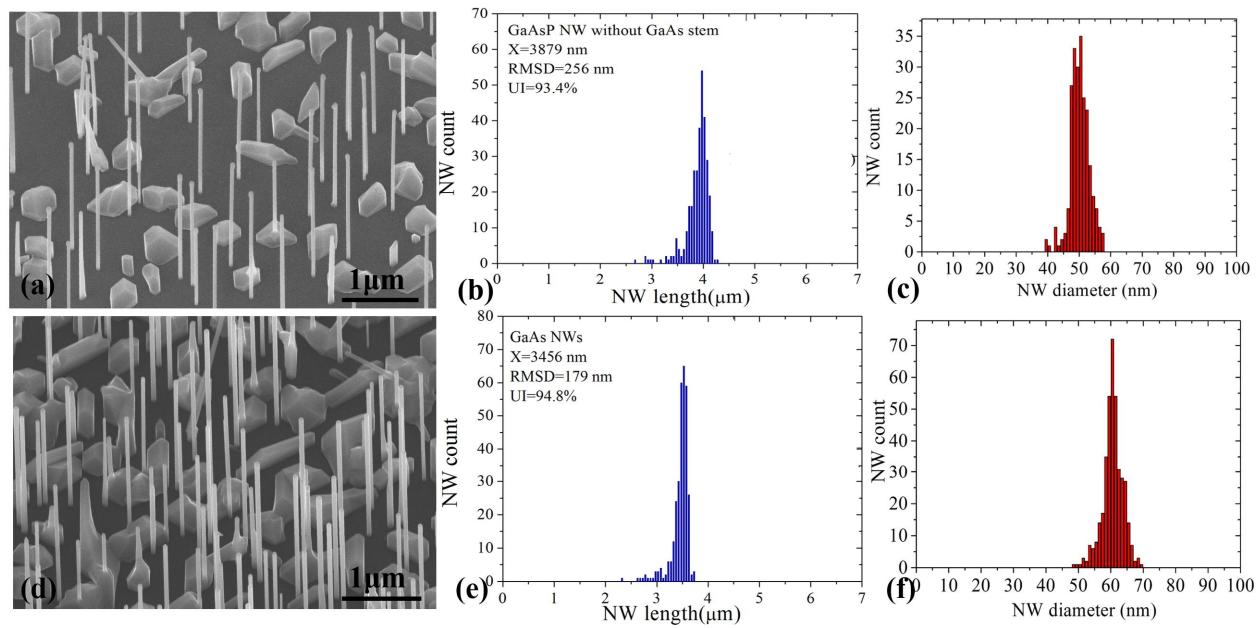

(c)

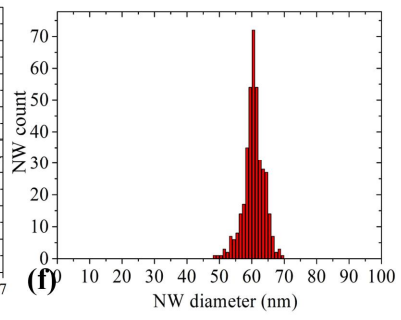

Figure 6. (a) $35^{\circ}$ tilted view SEM image, (b) length summation histogram and (c) diameter summation histogram of DG-GaAsP NWs. (d) $25^{\circ}$ tilted view SEM image, (e) length summation histogram and (f) diameter summation histogram of GaAs NWs.

According to above discussion, the droplet size distribution can greatly influence the NW uniformity. Thus, it is important to form uniform droplet size to achieve uniform NW growth. GaAsP NWs were grown without the GaAs stem and in addition, pure GaAs NWs were grown. There was no change in growth parameters during the growths to provide a comparatively stable environment, which is beneficial for formation of uniform droplet size. As can be seen in Figure 6, both GaAsP and GaAs NWs are highly uniform in length and diameter. The average NW length $(\bar{X})$, root mean square deviation (RMSD) and uniformity index (UI) are defined by the following equations:

$$
\bar{X}=\frac{1}{n} \sum_{i=6} X_{i}
$$




$$
\begin{aligned}
& \mathrm{RMSD}=\sqrt{\frac{1}{m} \sum_{t=1}^{n}\left(X_{t}-\bar{X}\right)^{2}} \\
& \mathrm{UI}=100 \% *(X-\mathrm{RM} 8 \mathrm{D}) / \bar{X}
\end{aligned}
$$

where the $\mathrm{n}$ is the number of NWs that were used in the summation. The UI is as high as $93.4 \%$ for GaAsP and $94.8 \%$ for GaAs NWs, which is in stark contrast with the GS-GaAsP NWs that is only $65.3 \%$.

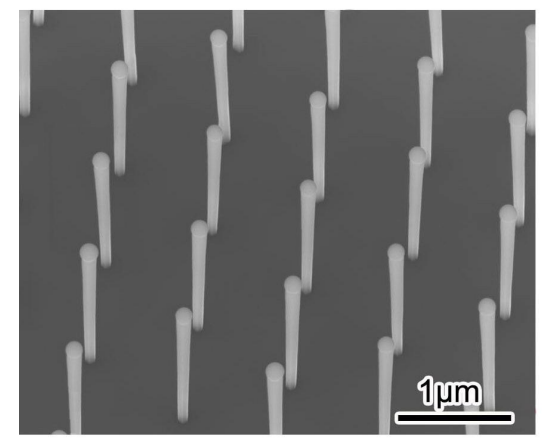

Figure 7. SEM images of GaAsP NWs grown on patterned Si substrates.

To achieve better droplet uniformity control, growths were performed on patterned Si substrates. ${ }^{23}$ The periodic pattern can make each NW has almost the same Ga collection area and hence droplet size. As can be seen in Figure 7, the NWs are highly uniform in length and diameter.

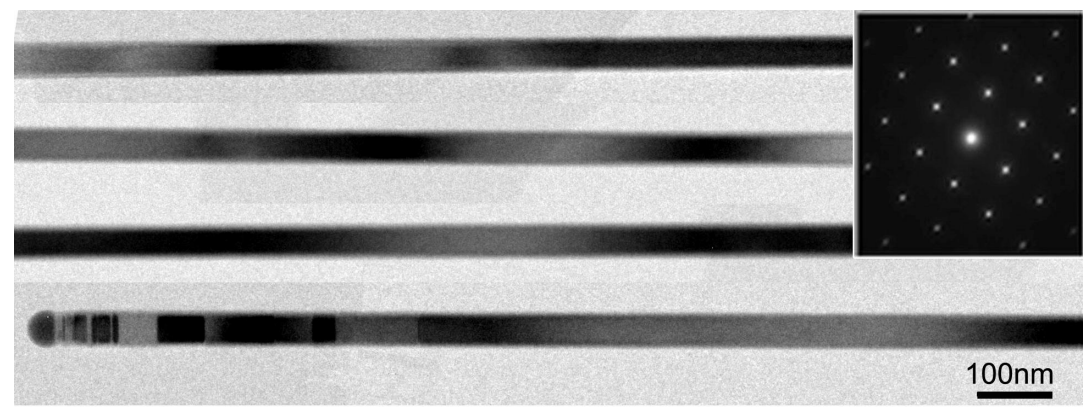

Figure 8. TEM images of a GaAsP NW. The inset is an electron diffraction pattern taken from the middle part of the NW.

The crystal quality of those NWs is shown in Figure 8. The body part is pure Zinc blend (ZB) and free of any defects. Defects are present at the very tip and very bottom parts, which is due to the fluctuation of growth environment and hence droplet properties at these stages.

\section{CONCLUSION}

In conclusion, the influences of the droplet size on growth of self-catalyzed ternary NWs were studied using GaAsP NWs. The NWs grown by droplets of smaller droplets can have slower growth rates and higher P content as compared with the larger ones. This is because nanoscale catalytic droplets are suffering from the GT effect, which makes smaller droplets having higher vapour pressure, lower supersaturation and hence slower nucleation rate. By controlling the size uniformity of the catalyst droplets, growth of both highly uniform and highly non-uniform NW heights have been demonstrated. These results not only give valuable information for understanding the NW nucleation mechanisms, but also provide additional knowledge on how to control composition and uniformity of the NWs. 


\section{REFERENCES}

[1] Zhang, Y., Wu, J., Aagesen, M., Liu, H., "III-V nanowires and nanowire optoelectronic devices," J. Phys. D: Appl. Phys., 48 (46), 463001 (2015).

[2] Lieber, C. M., Wang, Z. L., "Functional nanowires". Mrs Bulletin, 32(02), 99-108(2007).

[3]Yan, R., Gargas, D., \& Yang, P., "Nanowire photonics". Nature Photonics, 3(10), 569-576 (2009).

[4] Yang, P., Yan, R., Fardy, M., "Semiconductor nanowire: what's next? " Nano letters, 10(5), 1529-1536 (2010).

[5] Zhang, Y. Y., Yao, G. R. "Performance enhancement of blue light-emitting diodes with AlGaN barriers and a special designed electron-blocking layer". Journal of applied physics, 110(9), 093104 (2011).

[6] Zhang, Y. Y., Fan, G. H., Yin, Y. A., Yao, G. R. "Performance enhancement of blue light-emitting diodes without an electron-blocking layer by using special designed p-type doped InGaN barriers". Optics express, 20(101), A133A140 (2012).

[7] Zhang, Y. Y., Yin, Y. A. "Performance enhancement of blue light-emitting diodes with a special designed AlGaN/GaN superlattice electron-blocking layer". Applied physics letters, 99(22), 221103 (2011).

[8] Holm, J. V., Jørgensen, H. I., Krogstrup, P., Nygård, J., Liu, H., Aagesen, M., "Surface-passivated GaAsP singlenanowire solar cells exceeding 10\% efficiency grown on silicon". Nature communications, 4, 1498 (2013).

[9] Wu, J., Ramsay, A., Sanchez, A. M., Zhang, Y., Kim, D., Brossard, F. S. F., Salamo, G. G. (2015). "Defect-free selfcatalyzed GaAs/GaAsP nanowire quantum dots grown on silicon substrate". Nano Letters. Nano letters, 16(1), 504511(2016).

[10] Lee, A., Liu, H., Seeds, A., "Semiconductor III-V lasers monolithically grown on Si substrates". Semiconductor Science and Technology, 28(1), 015027(2013).

[11 ] Lee, A., Jiang, Q., Tang, M., Seeds, A., Liu, H., "Continuous-wave InAs/GaAs quantum-dot laser diodes monolithically grown on Si substrate with low threshold current densities". Optics express, 20(20), 2218122187(2012).

[12] Tang, M., Chen, S., Wu, J., Jiang, Q., Dorogan, V. G., Benamara, M., Mazur, Y. I., Salamo, G J., Seeds, A., Liu, H., "1.3- $\mu \mathrm{m}$ InAs/GaAs quantum-dot lasers monolithically grown on Si substrates using InAlAs/GaAs dislocation filter layers". Opt. Express, 22(10), 11528-11535 (2014).

[13] Wu, J., Lee, A., Jiang, Q., Tang, M., Seeds, A. J., Liu, H., "Electrically pumped continuous-wave 1.3- $\mu \mathrm{m}$ InAs/GaAs quantum dot lasers monolithically grown on Si substrates". IET Optoelectronics, 8(2), 20-24 (2014).

[14] Liu, H. Y., Steer, M. J., Badcock, T. J., Mowbray, D. J., Skolnick, M. S., Suárez, F., Ng, J. S., Hopkinson, M., David, J. P. R. (2006). "Room-temperature $1.6 \mu \mathrm{m}$ light emission from InAs/GaAs quantum dots with a thin GaAsSb cap layer". Journal of applied physics, 99, 046104.

[15] Liu, H. Y., Sellers, I. R., Badcock, T. J., Mowbray, D. J., Skolnick, M. S., Groom, K. M., Gutierrez, M., Hopkinson, M., Ng, J. S., David, J. P. R., Beanland, R., "Improved performance of 1.3 um multilayer InAs quantum-dot lasers using a high-growth-temperature GaAs spacer layer". Applied Physics Letters, 85, 704 (2004).

[16] Liu, H. Y., Hopkinson, M., Harrison, C. N., Steer, M. J., Frith, R., Sellers, I. R., Mowbray, D. J., Skolnick, M. S., "Optimizing the growth of $1.3 \mu \mathrm{m} \mathrm{InAs/InGaAs} \mathrm{dots-in-a-well} \mathrm{structure".} \mathrm{Journal} \mathrm{of} \mathrm{applied} \mathrm{physics,} \mathrm{93(5),} \mathrm{2931-}$ 2936 (2003).

[17] Liu, H. Y., Sellers, I. R., Gutierrez, M., Groom, K. M., Soong, W. M., Hopkinson, M., David, J. P. R., Beanland, R., Badcock, T. J., Mowbray, D. J., Skolnick, M. S., "Influences of the spacer layer growth temperature on multilayer InAs/GaAs quantum dot structures". Journal of applied physics, 96, 1988-1992 (2004).

[18] Zhang, Y., Sanchez, A. M., Sun, Y., Wu, J., Aagesen, M., Huo, S., Kim, D., Jurczak, P., Xu, X., Liu, H. "Influence of Droplet Size on the Growth of Self-Catalyzed Ternary GaAsP Nanowires". Nano letters, 16(2): 1237-1243 (2016).

[19] Zhang, Y., Aagesen, M., Holm, J. V., Jørgensen, H. I., Wu, J., Liu, H., "Self-catalyzed GaAsP nanowires grown on silicon substrates by solid-source molecular beam epitaxy". Nano letters, 13(8), 3897-3902 (2013).

[20] Zhang, Y., Sanchez, A. M., Wu, J., Aagesen, M., Holm, J. V., Beanland, R., Ward, T., Liu, H., Polarity-"Driven Quasi-3-Fold Composition Symmetry of Self-Catalyzed III-V-V Ternary Core-Shell Nanowires". Nano letters, 15(5), 3128-3133 (2015). 
[21] Wu, J., Li, Y., Kubota, J., Domen, K., Aagesen, M., Ward, T., Sanchez, A., Beanland, R., Zhang, Y., Tang, M., Hatch, S., Seeds, A., "Wafer-scale fabrication of self-catalyzed $1.7 \mathrm{eV} \mathrm{GaAsP}$ core-shell nanowire photocathode on silicon substrates". Nano letters, 14(4), 2013-2018 (2014).

[22] Tan, T. Y.; Li, N.; Gösele, U. "On the thermodynamic size limit of nanowires grown by the vapor-liquid-solid process" Applied Physics A, 78, 519-526 (2004).

[23] Zhang, Y., Wu, J., Aagesen, M., Holm, J., Hatch, S., Tang, M., Huo, S., Liu, H. (2014). "Self-Catalyzed Ternary Core-Shell GaAsP Nanowire Arrays Grown on Patterned Si Substrates by Molecular Beam Epitaxy". Nano letters, 14(8), 4542-4547. 